\title{
Introduzione alla conoscenza della sottofamiglia Aleocharinae della Guyana Francese: Parte IV ${ }^{1}$ (Coleoptera, Staphylinidae)
}

Con 70 figure

ROBERTO PACE ${ }^{2}$

${ }^{1} 287^{\circ}$ "Contributo alla conoscenza delle Aleocharinae".

${ }^{2}$ Via Vittorio Veneto, 13, 37032 Monteforte d’Alpone (Verona), Italia. - pace.ent@tiscali.it

Published on 2015-06-30

\section{Summary}

Description of 19 new species of the tribe Lomechusini (Part I): Orphnebius spinominutus spec. nov., Orphnebius guyanensis spec. nov., Orphnebius struyvei spec. nov., Orphnebius dentapicalis spec. nov., Orphnebius perinsolitus spec. nov., Orphnebius guysimplex spec. nov., Orphnebius diversipennis spec. nov., Orphnebius guylongipes spec. nov., Orphnebius carinapicalis spec. nov., Orphnebius curteparamericus spec. nov., Orphnebius microniger spec. nov., Tetradonia guyanensis spec. nov., Tetradonia anteopaca spec. nov., Tetradonella guyanorum spec. nov., Aychenonida guyanensis gen. nov., spec. nov., Macrogerodonia struyvei spec. nov., Macrogerodonia inina spec. nov., Euryepiskiosa crassa gen. nov., spec. nov., Euryepiskiosa guyanensis spec. nov. The new genera are Aychenonida gen. nov. and Euryepiskiosa gen. nov. The new species and new genera are illustrated and compared with similar species or genera.

\section{Key words}

Coleoptera, Staphylinidae, Aleocharinae, taxonomy, French Guyana

\section{Zusammenfassung}

Neunzehn neue Arten der Tribus Lomechusini (Teil I) werden beschrieben: Orphnebius spinominutus spec. nov., Orphnebius guyanensis spec. nov., Orphnebius struyvei spec. nov., Orphnebius dentapicalis spec. nov., Orphnebius perinsolitus spec. nov., Orphnebius guysimplex spec. nov., Orphnebius diversipennis spec. nov., Orphnebius guylongipes spec. nov., Orphnebius carinapicalis spec. nov., Orphnebius curteparamericus spec. nov., Orphnebius microniger spec. nov., Tetradonia guyanensis spec. nov., Tetradonia anteopaca spec. nov., Tetradonella guyanorum spec. nov., Aychenonida guyanensis gen. nov., spec. nov., Macrogerodonia struyvei spec. nov., Macrogerodonia inina spec. nov., Euryepiskiosa crassa gen. nov., spec. nov., Euryepiskiosa guyanensis spec. nov. Die neuen Gattungen sind: Aychenonida gen. nov. und Euryepiskiosa gen. nov. Neue Arten und neue Gattungen werden illustriert und mit ähnlichen Arten bzw. Gattungen verglichen.

\section{Riassunto}

Descrizione di 19 nuove specie della tribù Lomechusini (Parte I): Orphnebius spinominutus spec. nov., Orphnebius guyanensis spec. nov., Orphnebius struyvei spec. nov., Orphnebius dentapicalis spec. nov., Orphnebius perinsolitus spec. nov., Orphnebius guysimplex spec. nov., Orphnebius diversipennis spec. nov., Orphnebius guylongipes spec. nov., 
Orphnebius carinapicalis spec. nov., Orphnebius curteparamericus spec.nov., Orphnebius microniger spec. nov., Tetradonia guyanensis spec. nov., Tetradonia anteopaca spec. nov., Tetradonella guyanorum spec. nov., Aychenonida guyanensis gen. nov., spec. nov., Macrogerodonia struyvei spec. nov., Macrogerodonia inina spec. nov., Euryepiskiosa crassa gen. nov., spec. nov., Euryepiskiosa guyanensis spec. nov. I nuovi generi sono Aychenonida gen. nov. ed Euryepiskiosa gen. nov. Le nuove specie e i nuovi generi sono illustrati e comparati con specie o generi simili.

\section{Acronimi}

Gli olotipi e paratipi delle nuove specie e altro materiale sono conservati nei seguenti istituti:

FMNHC Field Museum of Natural History, Chicago IRSNB Institut royal des Sciences naturelles de Belgique, Bruxelles

MNHUB Museum für Naturkunde der HumboldtUniversität, Berlin

SDEI Senckenberg Deutsches Entomologisches Institut, Müncheberg

\section{LOMECHUSINI Parte prima}

\section{Orphnebius spinominutus spec. nov. \\ (Figg. 1 e 20-21)}

Materiale tipico: Holotypes ơ , Guyane Fr., Barrage Petit Saut, 10.XI.2011, leg. T. Struyve (IRSNB).

Descrizione: Lunghezza $1,7 \mathrm{~mm}$. Corpo lucido e giallo-rossiccio, capo nero, antenne rossicce con i due antennomeri basali giallo-rossicci, zampe giallo-rossicce con metà basale dei femori gialla. Occhi enormi. Secondo antennomero più corto del primo, terzo più corto del secondo, quarto a decimo trasversi. Reticolazione di capo ed elitre superficiale, quella di pronoto e addome evidente. Punteggiatura del capo fine e poco visibile. Granulosità del pronoto saliente, quella delle elitre fitta e saliente, quella dell'addome ridotta ad alcuni granuli. Quinto urotergo libero del maschio con tubercolo mediano posteriore saliente e schiacciato circolarmente sulla sommità. Edeago Figg. 20-21.

Comparazioni: Per la lunghezza dell'edeago, la nuova specie è simile a O. modestus PACE, 2009 del Brasile. Se ne distingue per la parte apicale dell'edeago, in visione ventrale, molto larga, mentre quella di modestus è molto stretta. Gli occhi della nuova specie sono molto più sviluppati di quelli di modestus.

Etimologia: La nuova specie prende nome dalla minuscola spina dell'armatura genitale interna dell'edeago.

\section{Orphnebius guyanensis spec. nov.}

(Figg. 2 e 22-24)

Materiale tipico: Holotypus $\sigma^{\star}$, Guyane Fr., Coralie, 16.XI.2011, leg. T. Struyve (SDEI).
Descrizione: Lunghezza $3 \mathrm{~mm}$. Corpo lucido e giallo-rossiccio, capo bruno, elitre bruno-rossicce, antenne e zampe giallo-rossicce. Occhi enormi. Secondo antennomero più corto del primo, terzo lungo quanto il secondo, quarto lungo quanto largo, quinto a decimo trasversi. Reticolazione del corpo invisibile. Punteggiatura fine solo presso gli occhi. Pronoto con solo due forti punti discali, sul resto della superficie la punteggiatura invisibile. Punteggiatura delle elitre irregolarmente distribuita e assente verso la sutura. Addome con qualche punto. Quinto urotergo libero del maschio con due solchi laterali e deboli pliche longitudinali presso il margine posteriore. Edeago Figg. 22-23, sesto urotergo libero del maschio Fig. 24.

Comparazioni: L’edeago della nuova specie è simile, in visione laterale, a quello di O. peruvianus PACE, 1986 del Perù, ma l'apice dell'edeago della nuova specie, in visione ventrale, è incavato, mentre quello di peruvianus è stretto, senza incavatura. Gli occhi della nuova specie sono più sviluppati di quelli di peruvianus.

Etimologia: La nuova specie prende nome dalla Guyana Francese.

\section{Orphnebius struyvei spec. nov.}

(Figg. 3 e 25)

Materiale tipico: Holotypus + , Guyane Fr., Coralie, 15.XI.2011, leg. T. Struyve (IRSNB).

Descrizione: Lunghezza 1,9 $\mathrm{mm}$. Corpo lucido e giallo-rossiccio, capo bruno-rossiccio, angoli posteriori esterni delle elitre sfumati di bruno, antenne giallo-rossicce con i due antennomeri basali gialli, zampe giallo-rossicce con femori gialli. Occhi enormi. Secondo antennomero più corto del primo, terzo più corto del secondo, quarto e quinto lunghi quanto larghi, sesto a decimo trasversi. Reticolazione del corpo invisibile. Punteggiatura del capo sparsa e molto superficiale, quella del pronoto sparsa con due punti discali evidenti, quella delle elitre rada e fine, assente agli angoli posteriori esterni presso cui si trovano due forti punti. Punteggiatura dell'addome ridotta a qualche punto. Quinto urotergo libero della femmina con quattro deboli carene longitudinali presso il margine posteriore. Spermateca Fig. 25.

Comparazioni: La spermateca della nuova specie è simile a quella di $O$. importunus Erichson, 1840 della Colombia, 
di cui ho esaminato lolotipo femmina (MNHUB). Se ne distingue per la minore lunghezza della spermateca, $0,15 \mathrm{~mm}$, mentre quella di importunus misura $0,21 \mathrm{~mm}$. La parte prossimale della spermateca della nuova specie è piegata una sola volta, quella di importunus due volte. Gli occhi della nuova specie sono molto sviluppati, quelli di importunus sono poco più lunghi della regione postoculare, in visione dorsale.

Etimologia: La nuova specie è dedicata al suo raccoglitore Tim Struyve, entomologo e consulente di pianificazione di Mechelen, Fiandre, Belgio.

\section{Orphnebius dentapicalis spec. nov.}

(Figg. 4 e 26-27)

Materiale tipico: Holotypus $0^{\star}$, Guyane Fr., Saramaka, 13.XI.2011, leg. T. Struyve (SDEI).

Paratypus: $1 \sigma^{*}$, stessa provenienza (IRSNB).

Descrizione: Lunghezza 3,4 mm. Corpo lucido, capo e pronoto neri, elitre brune, addome giallo-rossiccio, antenne brune con i tre antennomeri basali e apice dell'undicesimo giallo-rossicci, zampe giallo-rossicce. Occhi più lunghi della regione postoculare, in visione dorsale. Secondo antennomero più corto del primo, terzo più lungo del secondo, quarto, ottavo e nono lunghi quanto larghi, quinto a settimo più lunghi che larghi, decimo trasverso. Reticolazione del corpo invisibile. Punteggiatura del capo evidente e assente sulla fascia longitudinale mediana. Punteggiatura del pronoto fine e ben visibile, due forti punti discali su una linea trasversa. Punteggiatura delle elitre fitta ed evidente. Granulosità marginale dell'addome saliente. Quinto urotergo libero del maschio con deboli pliche longitudinali marginali. Edeago Figg. 26-27.

Comparazioni: L'habitus della nuova specie è simile a quello di O. ecuadorensis PACE, 1997 dell'Ecuador. Lapice dell'edeago della nuova specie, in visione ventrale, è largo e lievemente incavato, quello di ecuadorensis all'apice è espanso e non incavato. Il sesto urotergo libero del maschio della nuova specie è trilobato al margine posteriore, quello di ecuadorensis è rettilineo.

Etimologia: La nuova specie prende nome dai pezzi copulatori apicali delledeago a forma di dente.

\section{Orphnebius perinsolitus spec. nov.}

(Figg. 5 e 28-30)

Materiale tipico: Holotypus ${ }^{\star}$, Guyane Fr., Saramaka, 13.XI.2011, leg. T. Struyve (IRSNB).

Paratypus: $1 \sigma^{x}$, stessa provenienza (SDEI).
Descrizione: Lunghezza $4,6 \mathrm{~mm}$. Specie mirmecofila. Corpo lucido. Avancorpo bruno, addome giallo-rossiccio, antenne brune con i tre antennomeri basali e l'undicesimo giallo-rossicci, zampe giallo-rossicce con metà basale dei femori gialla. Occhi più lunghi della regione postoculare, in visione dorsale. Secondo antennomero più corto del primo, terzo più lungo del secondo, quarto a decimo più lunghi che larghi. Reticolazione del corpo invisibile. Punteggiatura del capo fitta e molto superficiale, quella dell'addome fine e molto evanescente. Granulosità del pronoto fine e fitta, invisibile all'indietro, con quattro forti punti in quadrato. Granulosità delle elitre fitta, forte e molto saliente. Quinto urotergo libero del maschio con un solco a ciascun lato e un callo mediano posteriore. Edeago Figg. 28-29, sesto urotergo libero del maschio Fig. 30.

Comparazioni: L’edeago della nuova specie è simile a quello di O. rioensis PACE, 2009 dell'Argentina. Lapice dell'edeago della nuova specie, in visione ventrale, è tronco, quello di rioensis arcuato. I penultimi antennomeri della nuova specie sono più lunghi che larghi, quelli di rioensis trasversi

Etimologia: La nuova specie ha nome che significa "Molto insolito" a causa della presenza della capsula dorsale dell'edeago molto sviluppata.

\section{Orphnebius guysimplex spec. nov.}

(Figg. 6 e 31-32)

Materiale tipico: Holotypus ${ }^{\star}$, Guyane Fr., Saramaka, 13.XI.2011, leg. T. Struyve (SDEI).

Descrizione: Lunghezza $4 \mathrm{~mm}$. Specie mirmecofila. Corpo lucido e bruno, capo nero, antenne giallo-rossicce. Occhi enormi, in visione dorsale. Secondo antennomero più corto del primo, terzo più lungo del secondo, quarto a decimo più lunghi che larghi. Reticolazione del corpo invisibile. Punteggiatura del capo fine e sparsa. Granulosità del pronoto fine e invisibile all'indietro, due forti punti mediani anteriori e due simili discali. Granulosità delle elitre molto saliente, quella dell'addome confinata, con alcuni granuli, al margine posteriore degli uroterghi liberi. Quinto urotergo libero del maschio con un solco a ciascun lato e una debole carena mediana. Edeago Figg. 31-32.

Comparazioni: Ledeago della nuova specie ha alcuni caratteri simili a quelli dell'edeago di O. loretensis PACE, 2008 del Perù. Se ne distingue per l'apice dell'edeago, in visione ventrale, incavato, mentre quello di loretensis è sporgente. Gli occhi della nuova specie sono molto grandi, quelli di loretensis poco sviluppati.

Etimologia: La nuova specie prende nome dalla struttura semplice o poco complessa delledeago. 


\section{Orphnebius diversipennis spec. nov.}

(Figg. 7 e 33-34)

Materiale tipico: Holotypus $\sigma^{\star}$, Guyane Fr., Saramaka, 13.XI.2011, leg. T. Struyve (IRSNB).

Descrizione: Lunghezza $4,5 \mathrm{~mm}$. Specie mirmecofila. Corpo lucido, capo nero, pronoto ed elitre bruni, addome giallo-rossiccio, antenne e zampe giallo-rossicce. Occhi enormi, in visione dorsale. Secondo antennomero più corto del primo, terzo più lungo del secondo, quarto a decimo più lunghi che larghi. Reticolazione del capo forte, quella del resto del corpo invisibile. Granulosità del capo superficiale, quella di pronoto ed elitre saliente e poco fitta, quella dell'addome composta di granuli lunghi posti al margine posteriore di ciascun urotergo libero. Pronoto con quattro forti punti discali in quadrato. Elitre con debole carena laterale. Quinto urotergo libero del maschio con un solco a ciascun lato e una debole carena mediana. Edeago Figg. 33-34.

Comparazioni: L'edeago della nuova specie, in visione laterale, è simile a quello di O. orejonesorum PACE, 2008 dell'Ecuador, ma l'apice dell'edeago della nuova specie, in visione ventrale, è quasi tronco, quello di orejonesorum è ogivale. I penultimi antennomeri della nuova specie sono più lunghi che larghi, quelli di orejonesorum trasversi.

Etimologia: La nuova specie ha nome che significa "Dalle elitre differenti” per la presenza di una debole carena laterale delle elitre non presente in specie del medesimo genere.

\section{Orphnebius guylongipes spec. nov} (Figg. 8 e 35-36)

Materiale tipico: Holotypus $\sigma^{\star}$, Guyane Fr., Saramaka, 13.XI.2011, leg. T. Struyve (SDEI).

Descrizione: Lunghezza 4,3 $\mathrm{mm}$. Specie mirmecofila. Corpo lucido, ma quinto urotergo libero del maschio opaco. Corpo giallo-rossiccio, capo nero-bruno, antenne e zampe giallo-rossicce. Occhi enormi, in visione dorsale. Secondo antennomero più corto del primo, terzo più lungo del secondo, quarto a decimo molto più lunghi che larghi. Reticolazione del corpo invisibile, ma sul pronoto molto superficiale. Granulosità di capo e pronoto molto forte e assente su una stretta fascia longitudinale mediana di entrambi, granulosità delle elitre saliente e fitta. Paratergiti basali terminanti all'indietro con un'appendice che porta una setola apicale. Quinto urotergo libero del maschio coperto di strie longitudinali che danno un aspetto opaco alla superficie, assenti su una stretta fascia longitudinale mediana che è percorsa da una debole carena. Edeago Figg. 35-36.
Comparazioni: L'edeago della nuova specie è simile a quello di O. venezuelanus PACE, 2011 del Venezuela, ma la parte apicale dell'edeago della nuova specie, in visione ventrale, è larga, quella di venezuelanus è stretta.

Etimologia: La nuova specie ha nome che significa "Lunghe zampe della Guyana Francese".

\section{Orphnebius carinapicalis spec. nov.}

(Figg. 9 e 37-39)

Materiale tipico: Holotypus $\sigma^{\star}$, Guyane Fr., Coralie, 15.XI.2011, leg. T. Struyve (IRSNB).

Paratypi: $2 \sigma^{\star} \sigma^{\star}$, stessa provenienza (SDEI).

Descrizione: Lunghezza 3,3 mm. Corpo lucido e bruno, base dell'addome giallo-bruna, antenne brune, zampe gialle con metà distale dei femori giallo-bruna. Occhi lunghi quanto la regione postoculare, in visione dorsale. Secondo antennomero più corto del primo, terzo più lungo del secondo, quarto a settimo più lunghi che larghi, ottavo lungo quanto largo, nono e decimo trasversi. Reticolazione del corpo invisibile. Punteggiatura del capo fine e poco evidente. Granulosità del pronoto saliente solo sulla metà posteriore, quella delle elitre distinta, ma assente su una fascia laterale, quella dell'addome ridotta a qualche granulo marginale. Quinto urotergo libero del maschio con un solco a ciascun lato e con granuli longitudinali solo al margine posteriore. Edeago Figg. 37-38, sesto urotergo libero del maschio Fig. 39.

Comparazioni: Ledeago e il sesto urotergo libero del maschio della nuova specie sono simili a quelli di O. ecuadorensis PACE, 1997 dell'Ecuador. Se ne distingue per la sinuosità preapicale dell'edeago, in visione laterale, molto stretta, mentre è larga in ecuadorensis. Il lobo mediano del sesto urotergo libero del maschio della nuova specie è molto largo, mentre è stretto quello di ecuadorensis. Gli occhi della nuova specie sono poco sviluppati, quelli di ecuadorensis enormi.

Etimologia: La nuova specie deriva il suo nome dalle due carene apicali ventrali dell'edeago.

\section{Orphnebius curteparamericus spec. nov. \\ (Figg. 10 e 40-41)}

Materiale tipico: Holotypus $\sigma^{\star}$, Guyane Fr., Coralie, 15.XI.2011, leg. T. Struyve (SDEI).

Descrizione: Lunghezza 2,4 mm. Corpo lucido e bruno-rossiccio, base dell'addome e pigidio rossicci, antenne brune con i tre antennomeri basali di un giallo pallido, zampe giallo-rossicce. Occhi più lunghi della regione postoculare, in visione dorsale. Secondo antennomero più corto del primo, terzo più corto del secondo, 
quarto a decimo trasversi. Reticolazione del corpo invisibile. Punteggiatura del capo sparsa, ma assente sulla fascia longitudinale mediana, quella del pronoto ridotta a quattro punti allineati presso il margine anteriore, sul resto della superficie la punteggiatura è invisibile. Punteggiatura delle elitre irregolarmente distribuita e sparsa. Una plica omerale obliqua. Quinto urotergo libero del maschio con rilievi laterali e carene marginali posteriori, sesto urotergo libero del maschio con carena saliente a $\mathrm{U}$ e sua base con forte reticolazione. Parameri dell'edeago molto corti. Edeago Figg. 40-41.

Comparazioni: Ledeago della nuova specie è simile a quello di O. loretensis PACE, 2008 del Perù. In visione ventrale, l'apice dell'edeago della nuova specie è molto largo, quello di loretensis molto stretto. Gli antennomeri intermedi della nuova specie sono trasversi, in loretensis sono più lunghi che larghi.

Etimologia: La nuova specie ha nome che richiama la ridotta lunghezza dei parameri.

\section{Orphnebius microniger spec. nov.}

(Figg. 11 e 42)

Materiale tipico: Holotypus + , Guyane Fr., Barrage Petit Saut, 10.XI.2011, leg. T. Struyve (IRSNB).

Descrizione: Lunghezza 1,9 mm. Corpo lucido e nerobruno, capo e quarto urotergo libero neri, antenne nere con i tre antennomeri basali di un giallo pallido, zampe giallo-rossicce. Occhi più lunghi della regione postoculare, in visione dorsale. Secondo antennomero più corto del primo, terzo più corto del secondo, quarto a decimo molto trasversi. Reticolazione del corpo invisibile. Granulosità dell'avancorpo poco saliente e poco fitta, quella dell'addome visibile solo al margine posteriore di ciascun urotergo libero. Spermateca Fig. 42.

Comparazioni: La forma della spermateca della nuova specie è simile a quella di O. napoensis PACE, 2008 dell'Ecuador. Il bulbo distale della spermateca della nuova specie è senza introflessione apicale, quello di napoensis ha lunga introflessione apicale del bulbo distale. Gli antennomeri intermedi sono molto trasversi nella nuova specie, poco trasversi in napoensis.

Etimologia: La nuova specie ha nome che indica il suo colore nero del corpo e la sua ridotta taglia corporea.

Tetradonia guyanensis spec. nov.

(Figg. 12 e 43-46)

Materiale tipico: Holotypus $\sigma^{\star}$, Guyane Fr., Coralie, 16.XI.2011, leg. T. Struyve (SDEI).
Paratypi: $60^{\star} o^{\star}$ e 3 우, stessa provenienza (IRSNB, SDEI).

Descrizione: Lunghezza $7 \mathrm{~mm}$. Specie mirmecofila. Corpo lucido, capo, pronoto, lati esterni e interni delle elitre e addome bruno-rossicci, resto delle elitre rossiccio, antenne nere con i due antennomeri basali e base del terzo bruni, zampe giallo-rossicce. Occhi più lunghi della regione postoculare, in visione dorsale. Secondo antennomero più corto del primo, terzo più lungo del secondo, quarto a sesto più lunghi che larghi, settimo e ottavo lunghi quanto larghi, nono e decimo trasversi. Reticolazione dell'avancorpo forte, quella dell'addome molto trasversa evidente. Punteggiatura di capo e pronoto fitta e superficiale. Granulosità delle elitre fitta e saliente. Capo con solco mediano, pronoto con un'impressione laterale e due punti discali infossati. Secondo urotergo libero del maschio con un largo callo mediano saliente, quinto con rughe al margine posteriore, sesto concavo. Edeago Figg. 44-45, spermateca Fig. 43, sesto urotergo libero del maschio Fig. 46.

Comparazioni: Per la forma della spermateca la nuova specie è simile a T. marginalis REICHENSPERGER, 1935 della Costa Rica, come illustrato da JACOBSON \& Kistner, 1998. Se ne distingue per il bulbo prossimale della spermateca piriforme e non sferico come in marginalis. Il sesto urotergo libero del maschio della nuova specie ha due lobi, quello di marginalis quattro.

Etimologia: La nuova specie prende nome dalla Guyana Francese.

\section{Tetradonia anteopaca spec. nov.}

(Figg. 13 e 47)

Materiale tipico: Holotypus + , Guyane Fr., Coralie, 16.XI.2011, leg. T. Struyve (IRSNB).

Descrizione: Lunghezza $5,7 \mathrm{~mm}$. Avancorpo molto opaco, addome lucido. Capo e pronoto neri, elitre e addome bruni, margine posteriore dei tre uroterghi liberi basali e pigidio rossicci, antenne nere con antennomero basale bruno, zampe giallo-rossicce con femori sfumati di bruno-rossiccio. Occhi più lunghi della regione postoculare, in visione dorsale. Secondo antennomero più corto del primo, terzo più lungo del secondo, quarto a decimo più lunghi che larghi. Reticolazione dell'avancorpo forte, quella dell'addome evidente, sugli uroterghi liberi quarto e quinto molto trasversa e ben visibile. Punteggiatura dell'avancorpo invisibile. Fronte senza reticolazione, capo e pronoto con debole e corto solco mediano. Spermateca Fig. 47.

Comparazioni: La spermateca della nuova specie ha alcuni caratteri simili a quelli della spermateca di T. hirsuta Jacobson \& Kistner, 1998 della Costa Rica. Se ne distin- 
gue per la parte prossimale molto più lunga di quella di hirsuta. I penultimi antennomeri della nuova specie sono più lunghi che larghi, quelli di hirsuta trasversi.

Etimologia: La nuova specie ha nome che indica che l'avancorpo è opaco.

\section{Tetradonella guyanorum spec. nov.}

(Figg. 14 e 48-50)

Materiale tipico: Holotypus $0^{*}$, Guyane Fr., Coralie, 16.XI.2011, leg. T. Struyve (SDEI).

Paratypi: 20 es., stessa provenienza (IRSNB, SDEI).

Descrizione: Lunghezza 3,9 mm. Avancorpo debolmente opaco, addome lucido. Avancorpo e uroterghi liberi quarto e quinto bruni, resto dell'addome giallo-rossiccio, antenne brune con i due antennomeri basali e base del terzo giallo-rossicci, zampe giallo-rossicce. Occhi più lunghi della regione postoculare, in visione dorsale. Secondo antennomero più corto del primo, terzo più lungo del secondo, quarto a decimo trasversi. Reticolazione dell'avancorpo forte, quella dell'addome evidente e sul quinto urotergo libero molto trasversa, pure evidente. Punteggiatura del capo fitta e molto superficiale, quella del pronoto fitta ed evidente. Granulosità delle elitre fitta e poco evanescente. Pronoto con impressioni laterali e debole solco mediano. Edeago Figg. 48-49, spermateca Fig. 50.

Comparazioni: La spermateca della nuova specie è simile a quella di T. latiarea JACOBson \& Kistner, 1998 del Perù e Costa Rica. Se ne distingue per il bulbo prossimale della spermateca molto più sviluppato di quello di latiarea. Il pronoto della nuova specie è meno trasverso di quello di latiarea. L'undicesimo antennomero della nuova specie è lungo quasi quanto i due antennomeri precedenti riuniti, quello di latiarea è lungo quanto i tre antennomeri precedenti riuniti.

Etimologia: La nuova specie ha nome che significa "Dei Guyanesi”.

Aychenonida gen. nov.

(Figg. 15 e 51-56)

\section{Diagnosi:}

Il nuovo genere è distinto da Apalonia CAsEy, 1906 per i caratteri seguenti:

1. Collo largo, paraglosse poco prominenti, lacinia stretta, galea poco più lunga della lacinia, angoli anteriori del mento non o poco prolungati Apalonia CASEY

- Collo stretto, paraglosse molto prominenti, Fig. 54, lacinia larga, galea molto più lunga della lacinia, Fig. 56, angoli anteriori del mento prolungati, Fig. 55.

Aychenonida gen. nov.
Tipo del nuovo genere: Aychenonida guyanensis spec. nov.

Etimologia: Il nome femminile del nuovo genere è composto dai vocaboli greco-antichi aúxív = collo e óveı $\delta$ o $=$ scherno. Il collo è quello stretto del nuovo genere, lo scherno è quello che subisce l'entomologo superficiale che avrebbe attribuito la nuova specie ad Autalia LEACH, 1819.

\section{Aychenonida guyanensis spec. nov \\ (Figg. 15 e 51-56)}

Materiale tipico: Holotypus ${ }^{\star}$, Guyane Fr., Saramaka, 13.XI.2011, leg. T. Struyve (SDEI).

Paratypi: $2 \sigma^{x} o^{x}$, stessa provenienza; 1 ㅇ, Guyane Fr., Barrage Petit Saut, 10.XI.2011, leg. T. Struyve (IRSNB).

Descrizione: Lunghezza 2,1 mm. Corpo lucido e nerobruno, capo, pronoto e quarto urotergo libero neri, antenne brune con i due antennomeri basali e base del terzo giallo-rossicci, zampe gialle. Occhi più lunghi della regione postoculare, in visione dorsale. Secondo antennomero più corto del primo, terzo più corto del secondo, quarto a decimo trasversi. Reticolazione di capo e addome superficiale, quella del pronoto forte, quella delle elitre evidente. Granulosità di capo ed elitre fine e superficiale, quella del pronoto fitta ed evidente, quella dell'addome invisibile. Pronoto con tre impressioni posteriori. Edeago Figg. 51-52, spermateca Fig. 53.

Etimologia: La nuova specie prende nome dalla Guyana Francese.

\section{Macrogerodonia struyvei spec. nov. (Figg. 16 e 57-59)}

Materiale tipico: Holotypus $0^{\star}$, Guyane Fr., Saramaka, 13.XI.2011, leg. T. Struyve (IRSNB).

Paratypus: 1 , stessa provenienza (IRSNB).

Descrizione: Lunghezza $6,5 \mathrm{~mm}$. Corpo lucido e giallo-rossiccio, capo nero-bruno, antenne brune con i quattro antennomeri basali e apice dell'undicesimo giallo-rossicci, zampe giallo-rossicce. Occhi più lunghi della regione postoculare, in visione dorsale. Secondo antennomero più corto del primo, terzo più lungo del secondo, quarto a settimo più lunghi che larghi, ottavo a decimo lunghi quanto larghi. Antennomeri non appiattiti. Reticolazione del corpo invisibile. Punteggiatura del capo composta di punti ampi e superficiali, quella del pronoto forte e assente in unarea presso gli angoli anteriori e posteriori, quella delle elitre forte e fitta. Granulosità dei due uroterghi liberi basali fitta, quella del quarto molto sparsa, quella degli uroterghi liberi quinto e sesto ridotta ad alcuni granuli lungo il margine posteriore. Pronoto con debole 
solco mediano. Sesto urotergo libero del maschio con due carene smussate. Edeago Figg. 57-58, spermateca Fig. 59.

Comparazioni: La nuova specie per l'habitus e la forma della spermateca è simile a $M$. peruviana (BERNHAUER, 1908) di cui ho esaminato l'olotipo ㅇ․ (FMNHC). La spermateca della nuova specie è stretta, con parte prossimale rettilinea, quella di peruviana robusta, con parte prossimale piegata ad angolo.

Etimologia: La nuova specie è dedicata al suo raccoglitore Tim Struyve, entomologo e consulente di pianificazione di Mechelen, Fiandre, Belgio.

\section{Macrogerodonia inina spec. nov. \\ (Figg. 17 e 60-61)}

Materiale tipico: Holotypus $\sigma^{\star}$, Guyane Fr., Coralie, 16.XI.2011, leg. T. Struyve (SDEI).

Paratypi: $2 \sigma^{\star} \sigma^{\star}$, stessa provenienza (IRSNB).

Descrizione: Lunghezza 4,5 mm. Corpo lucido e bruno-rossiccio, capo, pronoto e uroterghi liberi terzo a quinto bruni, antenne brune con i due antennomeri basali e metà basale del terzo giallo-rossicci, zampe gialle. Occhi più lunghi della regione postoculare, in visione dorsale. Secondo antennomero più corto del primo, terzo più lungo del secondo, quarto e quinto lunghi quanto larghi, sesto a decimo trasversi. Reticolazione delle elitre molto superficiale, quella del resto del corpo invisibile. Punteggiatura del capo fitta e superficiale, quella di pronoto ed elitre forte e fitta, sul pronoto assente su una stretta fascia longitudinale mediana. Primo urotergo libero del maschio con tre forti punti allineati trasversalmente e distanti dal margine posteriore, secondo urotergo libero del maschio con due punti forti allineati come sopra, terzo e quarto urotergo libero del maschio con due forti punti e granuli marginali, quinto urotergo libero del maschio con sei punti. Edeago Fig. 60, sesto urotergo libero del maschio Fig. 61.

Comparazioni: Per la forma del capo e delle elitre, la nuova specie è simile a $M$. magnicollis BernHAUER, 1941 del Perù, di cui ho esaminato l'olotipo maschio (FMNHC), esemplare privo di pronoto. Ledeago della nuova specie è tozzo, in visione laterale, con flagello interno, quello di magnicollis è stretto e senza flagello interno.

Etimologia: La nuova specie prende nome da Inini, territorio della Guyana Francese.

\section{Euryepiskiosa gen. nov.}

(Figg. 18 e 62-67)

Diagnosi: Il nuovo genere, per la forma delle parti orali è affine ad Apalonia CASEy, 1910. Se ne distingue per i caratteri della seguente chiave:
1. Lato anteriore del pronoto più largo del posteriore, addome non o poco ristretto all'indietro, lobi della ligula larghi e corti Apalonia CASEY

- Lato anteriore del pronoto più stretto del posteriore, addome ristretto all'indietro, lobi della ligula stretti e lunghi, Fig. 65 Euryepiskiosa gen. nov.

Tipo del nuovo genere: Euryepiskiosa crassa spec. nov.

Etimologia: Il nome femminile del nuovo genere, di derivazione dal greco antico, è composto da zúpúc = largo e $\dot{\varepsilon} \pi i \sigma \kappa ı \varsigma$ = opaco. Questi due caratteri sono quelli del corpo.

Chiave delle specie del nuovo genere Euryepiskiosa:

1. Pronoto più fortemente ristretto in avanti, regione postoculare sfuggente. Edeago Figg. 62-63, spermateca Fig. 64 E. crassa spec. nov.

- Pronoto meno fortemente ristretto in avanti, regione postoculare arrotondata. Edeago Figg. 68-69, spermateca Fig. 70 E. guyanensis spec. nov.

\section{Euryepiskiosa crassa spec. nov.}

(Figg. 18 e 62-67)

Materiale tipico: Holotypus $\sigma^{*}$, Guyane Fr., Caussade, 7.XI.2011, leg. T. Struyve (IRSNB).

Paratypus: 1 \% , stessa provenienza (SDEI).

Descrizione: Lunghezza 2,2 mm. Avancorpo debolmente opaco, addome lucido. Corpo giallo-rossiccio, capo e urotergo libero quarto bruni, elitre bruno-rossicce, antenne bruno-rossicce con i due antennomeri basali e metà apicale dell'undicesimo gialli, zampe giallo-rossicce. Occhi più lunghi della regione postoculare che è sfuggente, in visione dorsale. Secondo antennomero lungo quanto il primo, terzo più corto del secondo, quarto a decimo trasversi. Reticolazione dell'avancorpo superficiale, quella dell'addome molto trasversa ed evanescente. Granulosità di capo ed elitre molto fitta e saliente, quella del pronoto poco saliente, quella dell'addome confinata al margine posteriore di ciascun urotergo libero. Edeago Figg. 62-63, spermateca Fig. 64.

Etimologia: La nuova specie prende nome dal suo corpo tozzo.

\section{Euryepiskiosa guyanensis spec. nov. (Figg. 19 e 68-70)}

Materiale tipico: Holotypus $\sigma^{\star}$, Guyane Fr., Caussade, 7.XI.2011, leg. T. Struyve (IRSNB).

Paratypi: 7 es., stessa provenienza (IRSNB, SDEI). 
Descrizione: Lunghezza 2,4 mm. Avancorpo debolmente opaco, addome lucido. Corpo giallo-rossiccio, capo bruno, uroterghi liberi quarto e base del quinto nero-bruni, antenne giallo-rossicce con i due antennomeri basali e l'undicesimo gialli, zampe giallo-rossicce. Occhi più lunghi della regione postoculare, in visione dorsale. Secondo antennomero più corto del primo, terzo lungo quanto il secondo, quarto a decimo trasversi. Reticolazione dell'avancorpo forte, quella dell'addome molto trasversa e molto superficiale. Granulosità di capo ed elitre evanescente, quella del pronoto poco saliente, quella dell'addome ridotta a granuli marginali. Edeago Figg. 68-69, spermateca Fig. 70.

Etimologia: La nuova specie prende nome dalla Guyana Francese.

\section{Ringraziamenti}

Per avermi affidato in studio il materiale oggetto del presente lavoro rivolgo i miei più cordiali ringraziamenti al collega e naturalista belga Tim Struyve di Mechelen. Per il prestito di tipi ringrazio il Dr. A. Newton del Field Museum of Natural History di Chicago e il Dr. Manfred Uhlig del Museum für Naturkunde der Humboldt-Universität, Berlino.

\section{Bibliografia}

Bernhauer, M. 1908: Beitrag zur Staphylinidenfauna von Südamerika. - Archiv für Naturgeschichte 74: 283-372.

Bernhauer, M. 1941: Staphylinidae (Col.). - Beiträge zur Fauna Perus 1: 277-293.

CASEY, T. L. 1906: Observations on the Staphylinid groups Aleocharinae and Xantholinini chiefly of America. Transactions of the Academy of Science of St. Louis 16: $125-435$.

Erichson, W. F. 1840: Genera et Species Staphylinorum Insectorum Coleopterorum Familiae. Part 2: 401-954. - F.H. Morin, Berlin.

Jacobson, H. R. \& KistneR, D. H. 1998: A Redescription of the Myrmecophilous Genus Tetradonia and a Description of a New, Closely Related, Free-Living Genus, Tetradonella (Coleoptera: Staphylinidae). Sociobiology 31: 151-279.

Leach, W. 1819: New Genera. - In: Samouelle, G.: The Entomologist's useful Compendium: 1-496, London.

PACE, R. 1986: Aleocharinae del Perù (Coleóptera Staphylinidae). - Redia 69: 417-467.
PACE, R. 1997: Aleocharinae della Colombia e dell'Ecuador, Parte III (conclusione) (Coleoptera, Staphylinidae) $\left(133^{\circ}\right.$ Contributo alla conoscenza delle Aleocharinae). - Revue suisse de Zoologie 104: 17-48.

PACE, R. 2008: New records of Aleocharinae from Ecuador and Peru, with the description of new species, new subgenera and new genera (Coleoptera, Staphylinidae) pp. 225-398. - In: Giachino, P. M. (ed.) 2008: Biodiversity of South America I. Memoirs on Biodiversity, World Biodiversity Association onlus, Verona 1: $496 \mathrm{pp}$.

Pace, R. 2009: Nuovi dati faunistici e tassonomici su Aleocharinae delle tribù Lomechusini, Hoplandriini, Oxypodini e Aleocharini del Sudamerica (Coleoptera, Staphylinidae). - Beiträge zur Entomologie, Keltern 59 (1): 133-173.

PACE, R. 2011: New and additional records, new genera and new species of Aleocharinae from Venezuela, Ecuador and Peru (Coleoptera, Staphylinidae). Bollettino del Museo Civico di Storia Naturale di Verona, Botanica Zoologia 35: 43-70.

Reichensperger, A. 1935: Beitrag zur Kenntnis der Myrmekophilenfauna Brasiliens und Costa Ricas III. - Arbeiten der morphologische und taxonomische Entomologie 2: 188-218. 


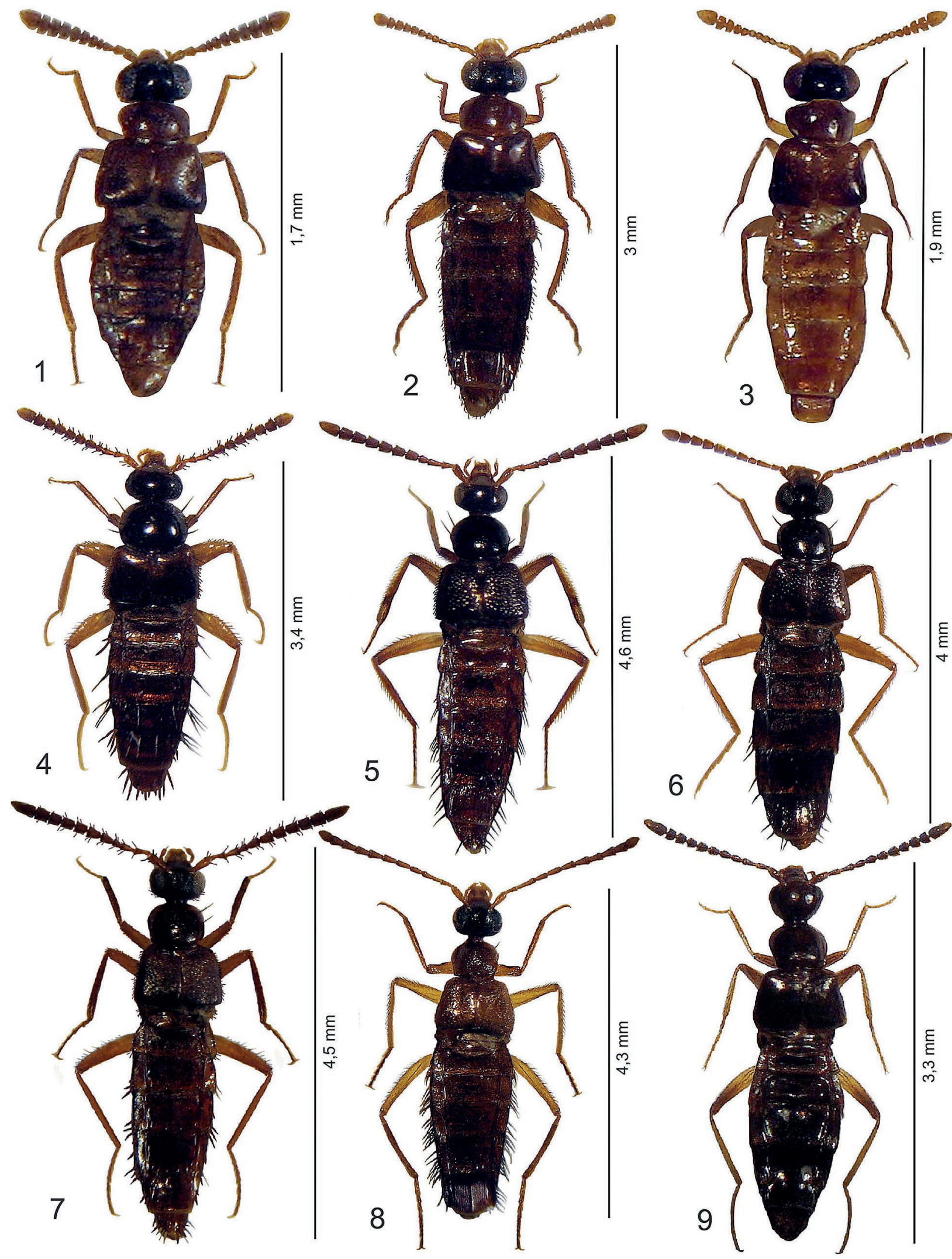

Figg. 1-9: Habitus. 1. Orphnebius spinominutus spec. nov.; 2. Orphnebius guyanensis spec. nov.; 3. Orphnebius struyvei spec. nov.; 4. Orphnebius dentapicalis spec. nov.; 5. Orphnebius perinsolitus spec. nov.; 6. Orphnebius guysimplex spec. nov.; 7. Orphnebius diversipennis spec. nov.; 8. Orphnebius guylongipes spec. nov.; 9. Orphnebius carinapicalis spec. nov. 

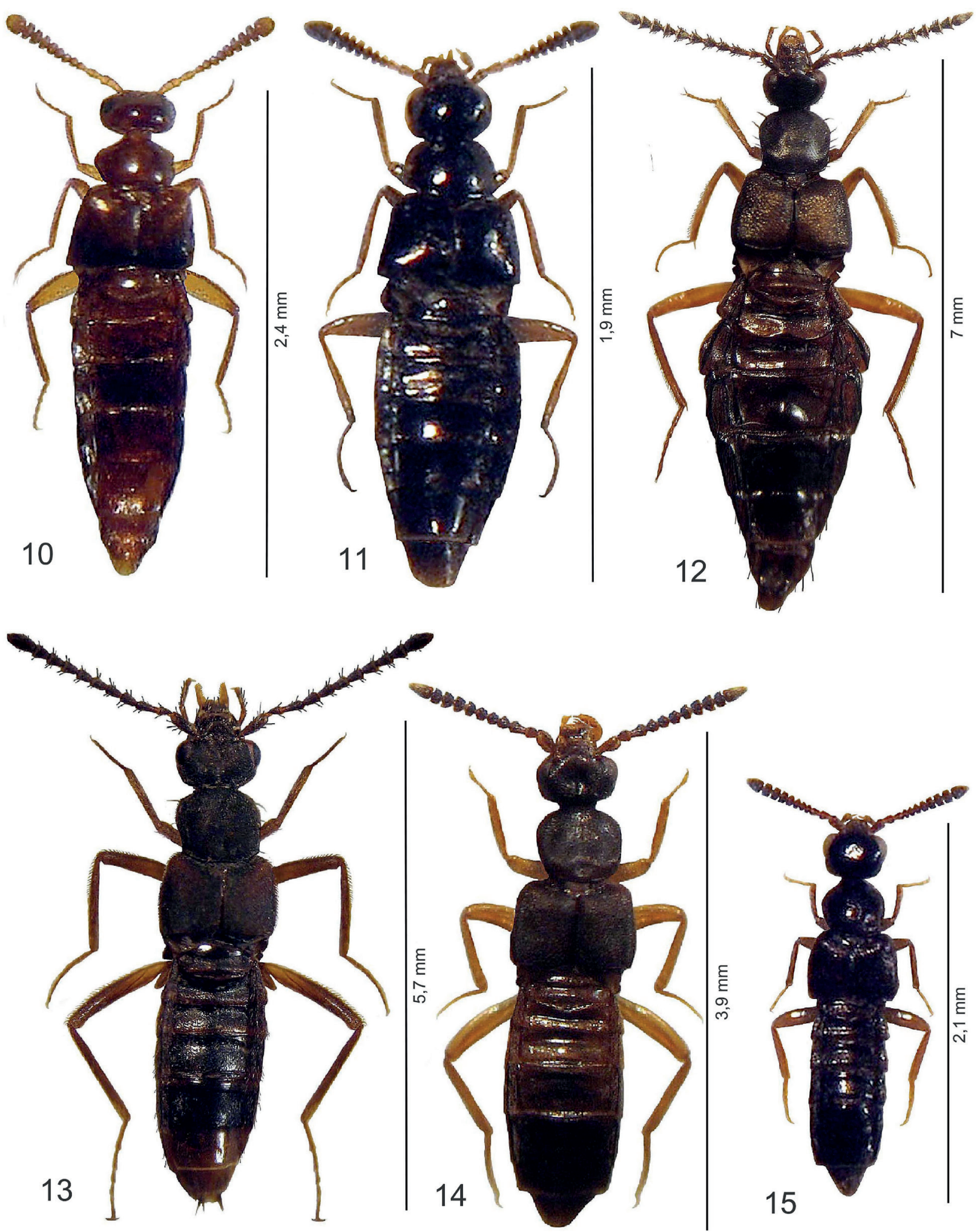

Figg. 10-15: Habitus. 10. Orphnebius curteparamericus spec. nov.; 11. Orphnebius microniger spec. nov.; 12. Tetradonia guyanensis spec. nov.; 13. Tetradonia anteopaca spec.nov.; 14. Tetradonella guyanorum spec. nov.; 15. Aychenonida guyanensis gen. nov., spec. nov. 


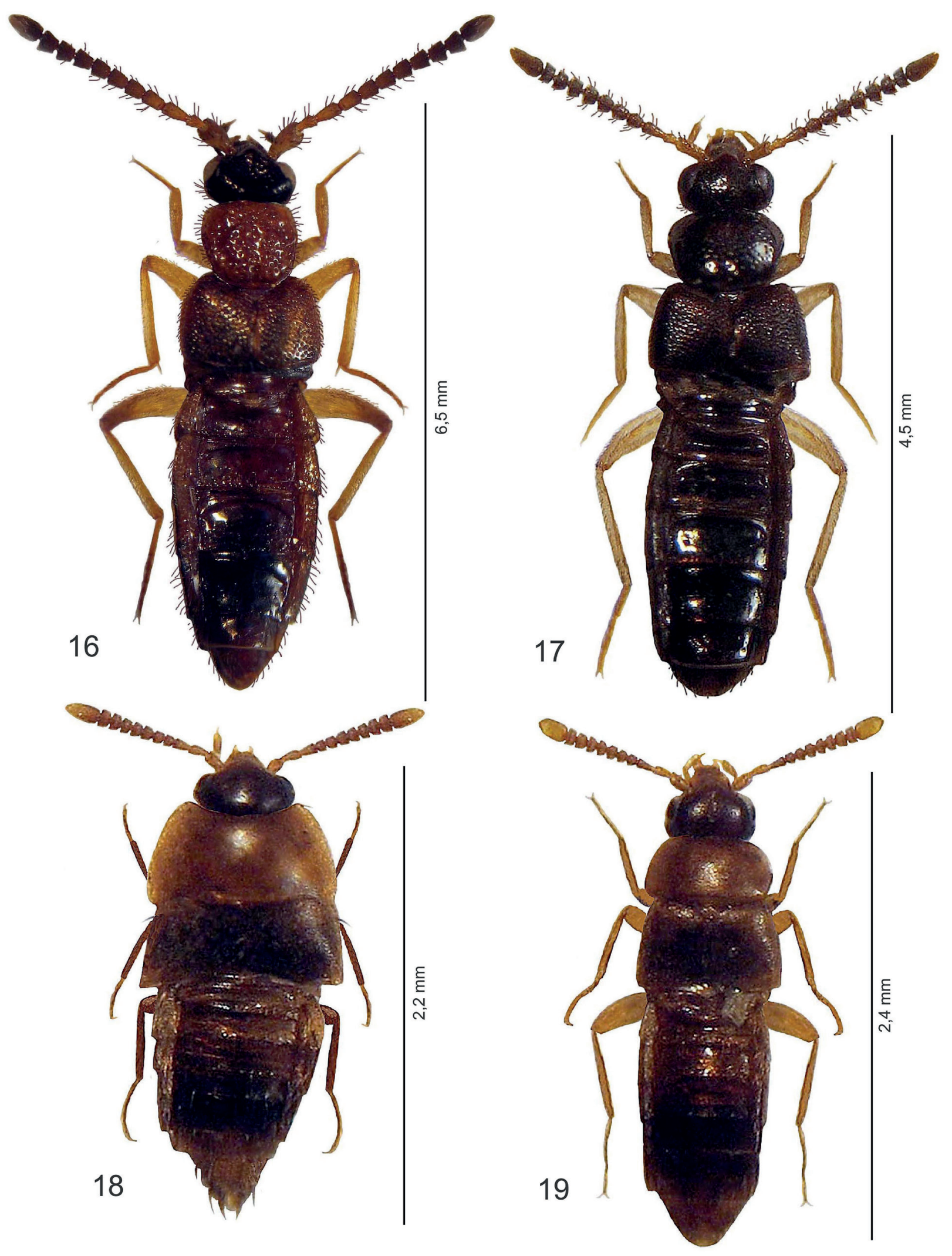

Figg. 16-19: Habitus. 16. Macrogerodonia struyvei spec. nov.; 17. Macrogerodonia inina spec. nov.; 18. Euryepiskiosa crassa gen. nov., spec. nov.; 19. Euryepiskiosa guyanensis gen. nov., spec. nov. 

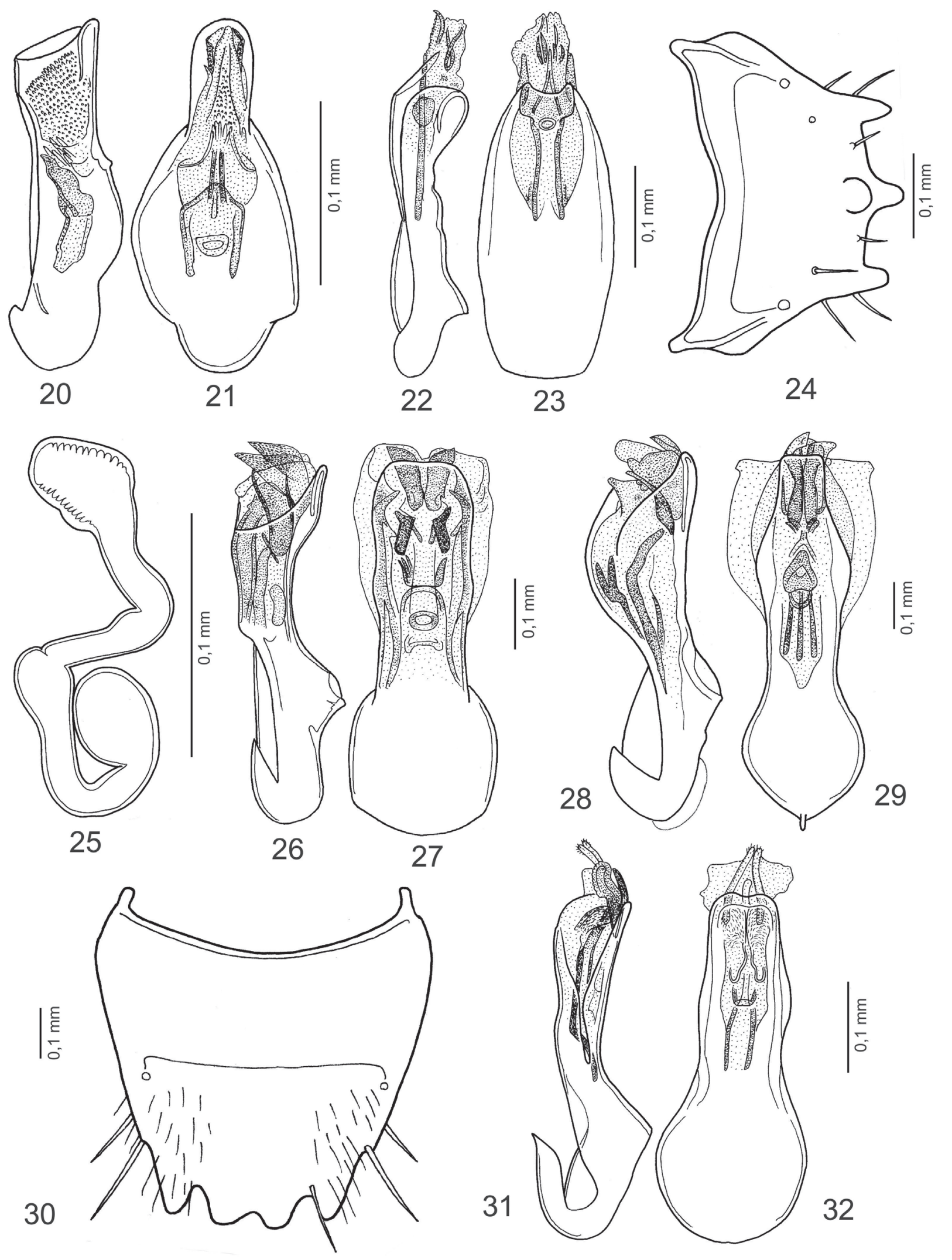

Figg. 20-32: Edeago in visione laterale e ventrale, sesto urotergo libero del maschio e spermateca. 20-21. Orphnebius spinominutus spec. nov.; 22-24. Orphnebius guyanensis spec. nov.; 25. Orphnebius struyvei spec. nov.; 26-27. Orphnebius dentapicalis spec. nov.; 28-30. Orphnebius perinsolitus spec. nov.; 31-32. Orphnebius guysimplex spec. nov. 

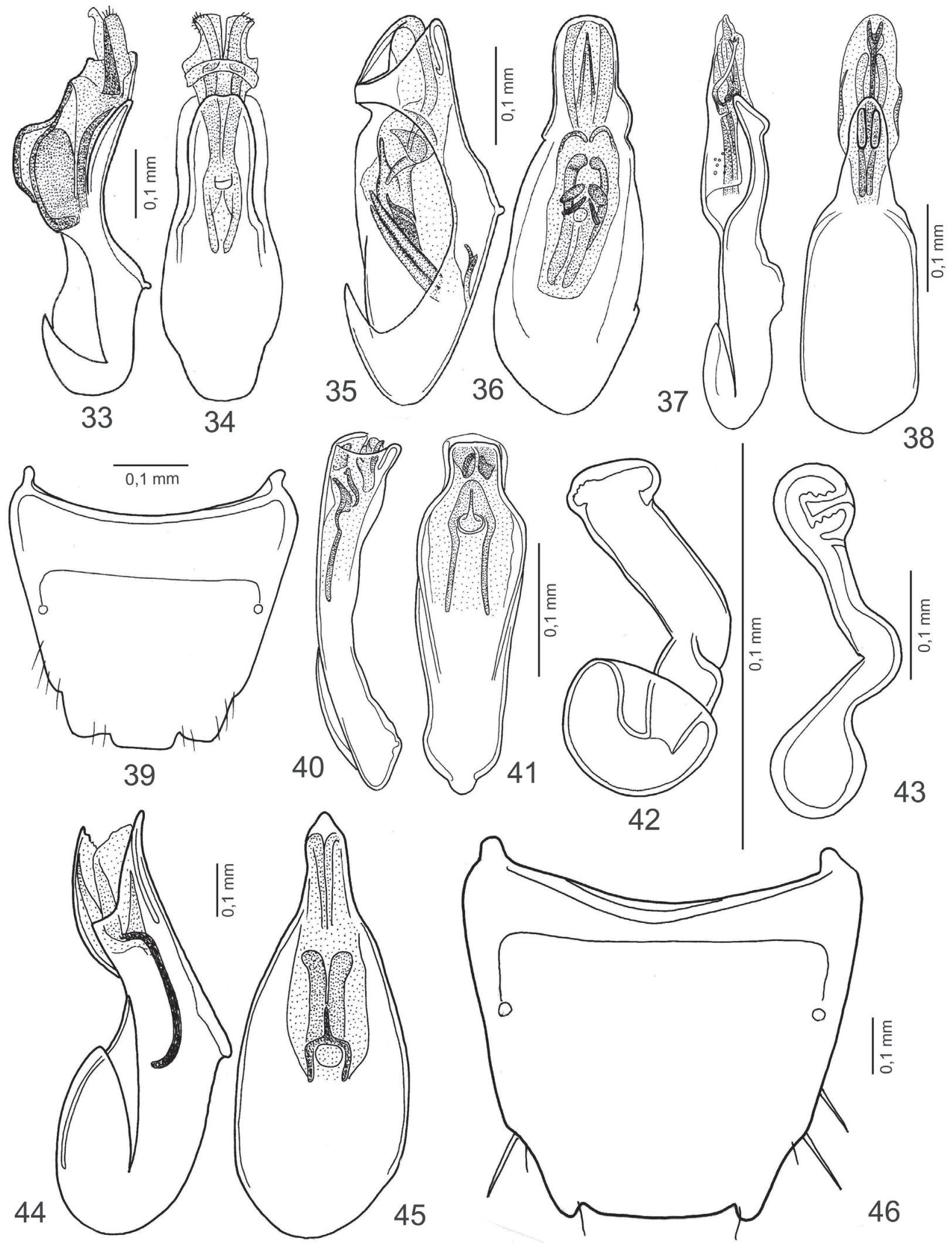

\section{3}

Figg. 33-46: Edeago in visione laterale e ventrale, sesto urotergo libero del maschio e spermateca. 33-34. Orphnebius diversipennis spec. nov.; 35-36. Orphnebius guylongipes spec. nov.;37-39. Orphnebius carinapicalis spec. nov.; 40-41. Orphnebius curteparamericus spec. nov.; 42. Orphnebius microniger spec. nov.; 43-46. Tetradonia guyanensis spec. nov. 

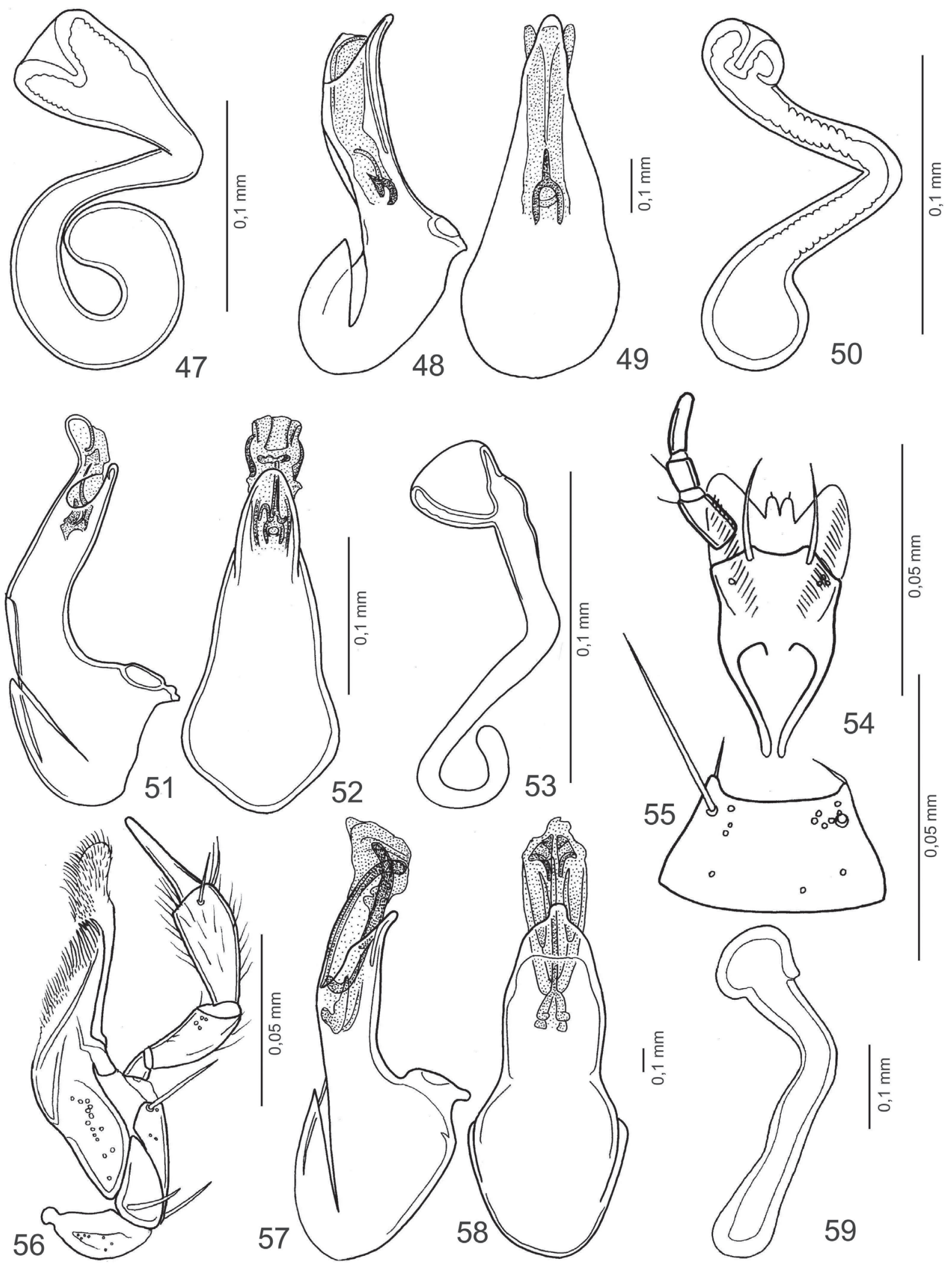

Figg. 47-59: Spermateca, edeago in visione laterale e ventrale, labio con palpo labiale, mento e maxilla con palpo mascellare. 47. Tetradonia anteopaca spec. nov.; 48-50. Tetradonella guyanorum spec. nov.; 51-56. Aychenonida guyanensis gen. nov., spec. nov.; 57-59. Macrogerodonia struyvei spec. nov. 

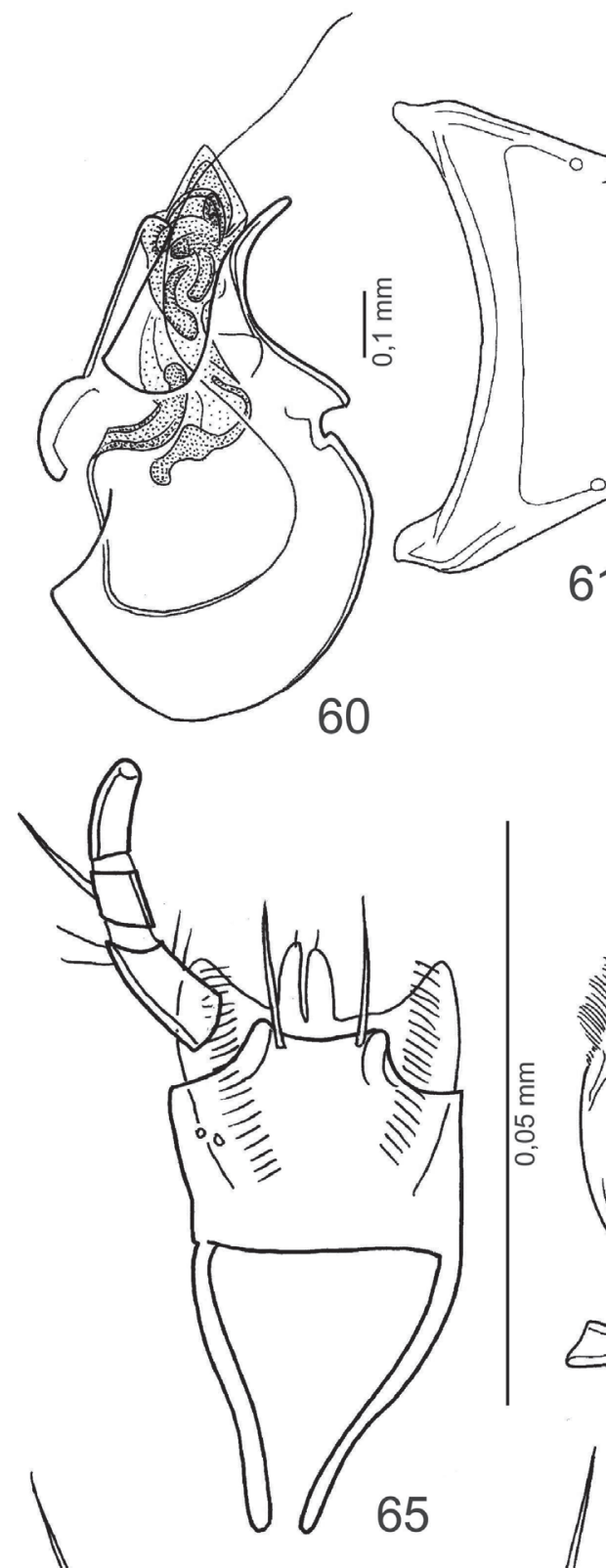

61
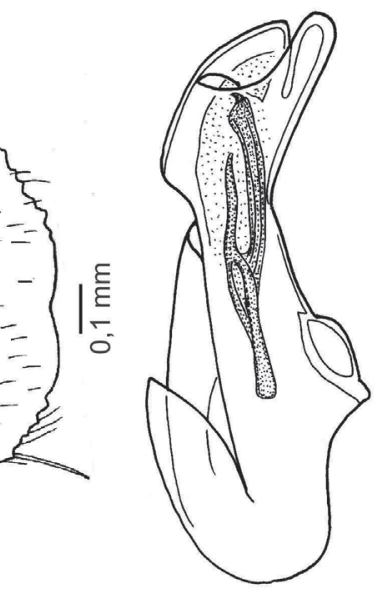

62

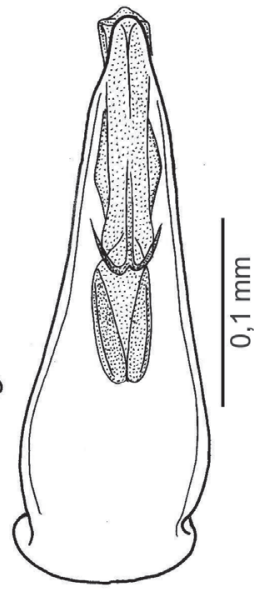

63
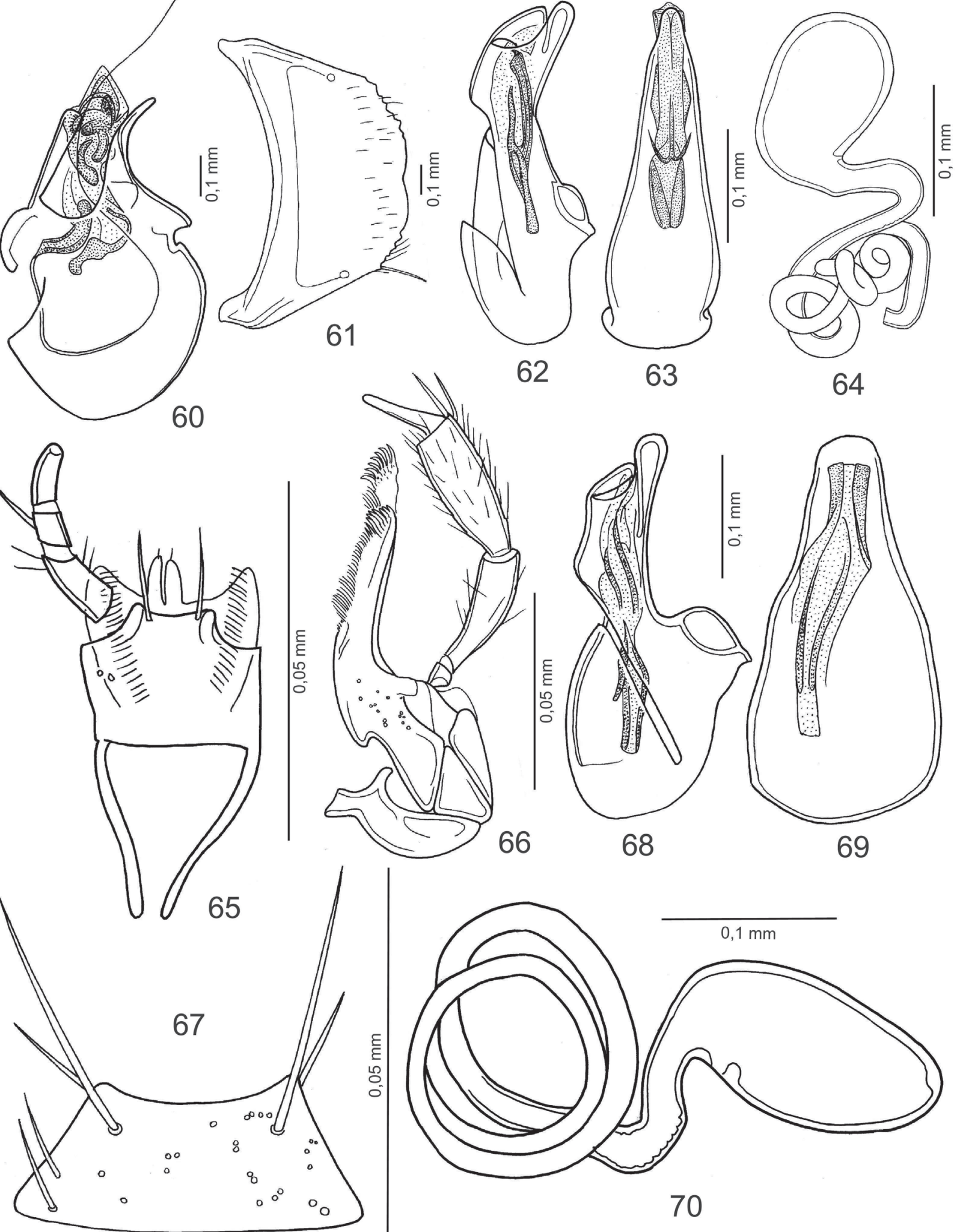

70

Figg. 60-70: Edeago in visione laterale e ventrale, sesto urotergo libero del maschio, labio con palpo labiale, mento e maxilla con palpo mascellare. 60-61. Macrogerodonia inina spec. nov.; 62-67. Euryepiskiosa crassa gen. nov., spec. nov.; 68-70. Euryepiskiosa guyanensis spec. nov. 